\title{
Bunyavirus transmis par les moustiques émergents au Canada
}

\author{
Drebot $\mathrm{MA}^{1^{*}}$ \\ ${ }^{1}$ Laboratoire national de microbiologie, Agence de la santé publique du Canada, Winnipeg, (Manitoba) \\ *Correspondance : mike.drebot@phac-aspc.gc.ca
}

\section{Résumé}

Les virus du sérogroupe California et le virus de Cache Valley sont des arbovirus (agents pathogènes transmis par les tiques et les moustiques) appartenant au genre Orthobunyavirus (famille Bunyaviridae). Bien que la majorité des expositions à ces virus entraînent des infections asymptomatiques ou bénignes, les virus du sérogroupe California et le virus de Cache Valley peuvent être à l'origine de maladies fébriles et neurologiques de nature similaire à celles associées aux infections par le virus du Nil occidental. Les virus du sérogroupe California et le virus de Cache Valley sont répartis partout en Amérique du Nord et circulent dans un certain nombre d'hôtes vertébrés et de moustiques porteurs, y compris plusieurs espèces de moustiques Aedes et d'autres moustiques n'appartenant pas au genre Culex. Le virus de Jamestown Canyon et le virus snowshoe hare sont la sorte la plus commune de virus du sérogroupe California au Canada et on en a relevé dans tout le pays. Ces agents pathogènes potentiels peuvent contribuer à un fardeau de la maladie plus élevé que celui reconnu auparavant et doivent être pris en compte dans le cadre du diagnostic différentiel des maladies fébriles et neuro-invasives pendant la saison des moustiques. Le diagnostic peut être fait en demandant un panel de diagnostic au niveau du programme de lutte contre les zoonoses virales au Laboratoire national de microbiologie. Afin de réduire le risque d'infection, il faut informer le public au sujet de ces virus et souligner l'importance de la prise de mesures de prévention personnelles.

\section{Introduction}

La famille Bunyaviridae de virus à ARN constitue un groupe très important, diversifié et réparti à l'échelle mondiale de virus qui infectent les plantes, les vertébrés et les invertébrés (1). De nombreux bunyavirus importants sur le plan médical sont des virus à transmission vectorielle qui peuvent infecter les rongeurs ou les arthropodes. Par exemple, les hantavirus tels que le virus Sin Nombre sont transmis par la souris sylvestre et entraînent un syndrome pulmonaire à hantavirus $(2,3)$. Les arbovirus sont des bunyavirus qui infectent et sont transmis au moyen des tiques et des moustiques. Les bunyavirus transmis par les moustiques appartiennent au genre Orthobunyavirus. Ce genre comprend environ 170 virus : 48 espèces et 19 sérogroupes. Dans deux de ces sérogroupes se trouvent quatre virus émergents qui sont de plus en plus reconnus comme des agents pathogènes pour les humains et les animaux (1). (Tableau explicatif)

\section{Quatre arbovirus émergents en Amérique du Nord appartenant au genre Orthobunyavirus de la famille Bunyaviridae}

Sérogroupe California : Ce sérogroupe comprend 17 virus notamment les suivants :

Virus de l'encéphalite de Californie

Virus d'Inkoo

Virus de Jamestown Canyon

Virus de La Crosse

Virus snowshoe hare

Virus de Tahyna

Sérogroupe Bunyamwera : Ce sérogroupe comprend 23 virus, notamment le suivant :

- Virus de Cache Valley

\footnotetext{
${ }^{1}$ Les quatre virus émergents sont indiqués en caractères gras.
} 
Les virus du sérogroupe California circulent partout dans le monde. Ils comprennent le virus d'Inkoo en Europe; le virus de Tahyna en Europe, en Asie et en Afrique; et le virus de La Crosse, le virus snowshoe hare et le virus de Jamestown Canyon en Amérique du Nord $(4,5)$. Plusieurs virus du sérogroupe California sont associés à des maladies pseudogrippales bénignes et à des infections graves du système nerveux central $(4,6,7,8)$. Le virus snowshoe hare a été mis en cause dans des cas neurologiques touchant principalement des enfants $(4,8,9)$. Parmi les virus du sérogroupe California étendus, on trouve également le virus de Jamestown Canyon qui a été récemment identifié comme une cause émergente de maladie neuro-invasive et fébrile au Canada et aux États-Unis $(6,7,8,10,11,12)$. Le virus de La Crosse est étroitement lié au virus snowshoe hare et est la cause principale d'encéphalite virale chez les enfants aux États-Unis (en moyenne 80 à 100 cas par an) et la deuxième cause principale de maladie neuro-invasive associée à un arbovirus en Amérique du Nord $(6,13)$. Aucun cas clinique n'a été signalé pour le moment au Canada, vraisemblablement en raison de la fréquence limitée de ses vecteurs (p. ex. Aedes triseriatis et Aedes albopictus). Cependant, les changements climatiques futurs pourraient avoir une influence sur l'expansion de ces espèces de moustiques vers le nord (14).

Le quatrième Orthobunyavirus émergent transmis par les moustiques est le virus de Cache Valley (sérogroupe Bunyamwera) qui circule partout en Amérique du Nord et en Amérique du Sud $(15,16)$. II est principalement associé à des maladies du bétail, en particulier des moutons (15). Toutefois, des patients atteints d'une maladie non diagnostiquée dans l'Ouest du Canada se sont révélés porteurs d'anticorps propres au virus de Cache Valley (17, résultats non publiés de Drebot) et plusieurs cas de maladie neuro-invasive chez les humains causée par le virus de Cache Valley ont été relevés aux États-Unis $(18,19)$.

II n'existe pas de traitements précis ou de vaccins actuellement disponibles pour le sérogroupe California et les infections par le virus de Cache Valley. Le traitement de ces infections virales comprend généralement des soins de soutien et la prise en charge des complications, p. ex. le soulagement de la pression intracrânienne accrue.

Les effets de ces bunyavirus sur la santé pourraient être beaucoup plus importants que ce qu'on pensait auparavant. De 1989 à 2005, aucun cas d'infections par les virus du sérogroupe California n'a été relevé au Canada en raison de l'interruption des procédures de diagnostic permettant de repérer ces agents pathogènes. Dans le cadre d'une approche de perfectionnement des tests sérologiques pour le virus du Nil occidental et d'autres arbovirus transmis par les moustiques, de nouvelles méthodes de dépistage ont été mises en œuvre et ajoutées aux panels de diagnostic existants au niveau du programme de lutte contre les zoonoses virales au Laboratoire national de microbiologie de Winnipeg, au Manitoba $(11,12,20)$.

II existe maintenant des preuves suffisantes pour recommander que, lorsque des patients présentent une maladie fébrile et neurologique ainsi que des antécédents d'exposition aux moustiques, le virus du Nil occidental et les bunyavirus transmis par les moustiques soient recherchés. Dans le présent article, l'écologie, l'épidémiologie, les aspects cliniques, les diagnostics et certaines des récentes données de surveillance en laboratoire des virus du sérogroupe California et du virus de Cache Valley seront analysés.

\section{Virus du sérogroupe California}

Le virus de La Crosse, le virus snowshoe hare et le virus de Jamestown Canyon sont les principaux virus du sérogroupe California qui sont émergents et négligés en Amérique du Nord $(4,6,7,9,11,13)$. Les humains peuvent contracter l'infection par l'intermédiaire de piqûres de moustique à l'origine d'une maladie asymptomatique à légèrement fébrile (fièvre, frissons, douleurs abdominales, toux, céphalées et photophobie) et d'une infection aiguë du système nerveux central (méningite ou encéphalite). Bien que la plupart des patients atteints d'encéphalite virale du sérogroupe California se rétablissent complètement, certaines séquelles neurologiques de longue durée ont été relevées, en particulier pour le virus de La Crosse (13).

Au Canada, l'activité des virus du sérogroupe California a été démontrée dans toutes les provinces et tous les territoires $(8,9,11,12)$. De 1978 à 1989, 23 cas d'infection symptomatique ont été diagnostiqués au Canada. La majorité des cas était liée au virus snowshoe hare (18 cas), trois cas au virus de Jamestown Canyon et deux cas à des virus du sérogroupe California dont l'identité était inconnue (8, Artsob et Drebot, résultats non publiés). 
Le risque d'exposition aux virus du sérogroupe California s'étend de mai à octobre, étant donné que les vecteurs prédominants porteurs du virus snowshoe hare et du virus de Jamestown Canyon sont (contrairement au virus du Nil occidental) des moustiques n'appartenant pas au genre Culex tels que les espèces Aedes, Culiseta et Anopheles $(4,7,8)$. Ainsi, les hôtes amplificateurs ou réservoirs de ces virus sont de petits mammifères tels que les écureuils, les tamias, les lièvres et divers rongeurs (virus snowshoe hare), ou bien de plus gros animaux tels que les cerfs et les wapitis (virus de Jamestown Canyon) (Figure 1). Le bétail, comme les chevaux, les bovins et les moutons présentent aussi des taux de séroprévalence importants, toutefois, ils ne contribuent probablement pas de façon importante au cycle de transmission enzootique de ces virus en raison de la faible virémie (21, Drebot, résultats non publiés). La transmission transovarienne est le mécanisme d'hivernage le plus probable. Ce mécanisme implique des moustiques infectés transmettant le virus à leur progéniture dans l'œuf, où le virus snowshoe hare et le virus de Jamestown Canyon hivernent.

\section{Figure 1 : Cycles de transmission des virus du sérogroupe California (virus de Jamestown Canyon, virus snowshoe hare) et du virus de Cache Valley}

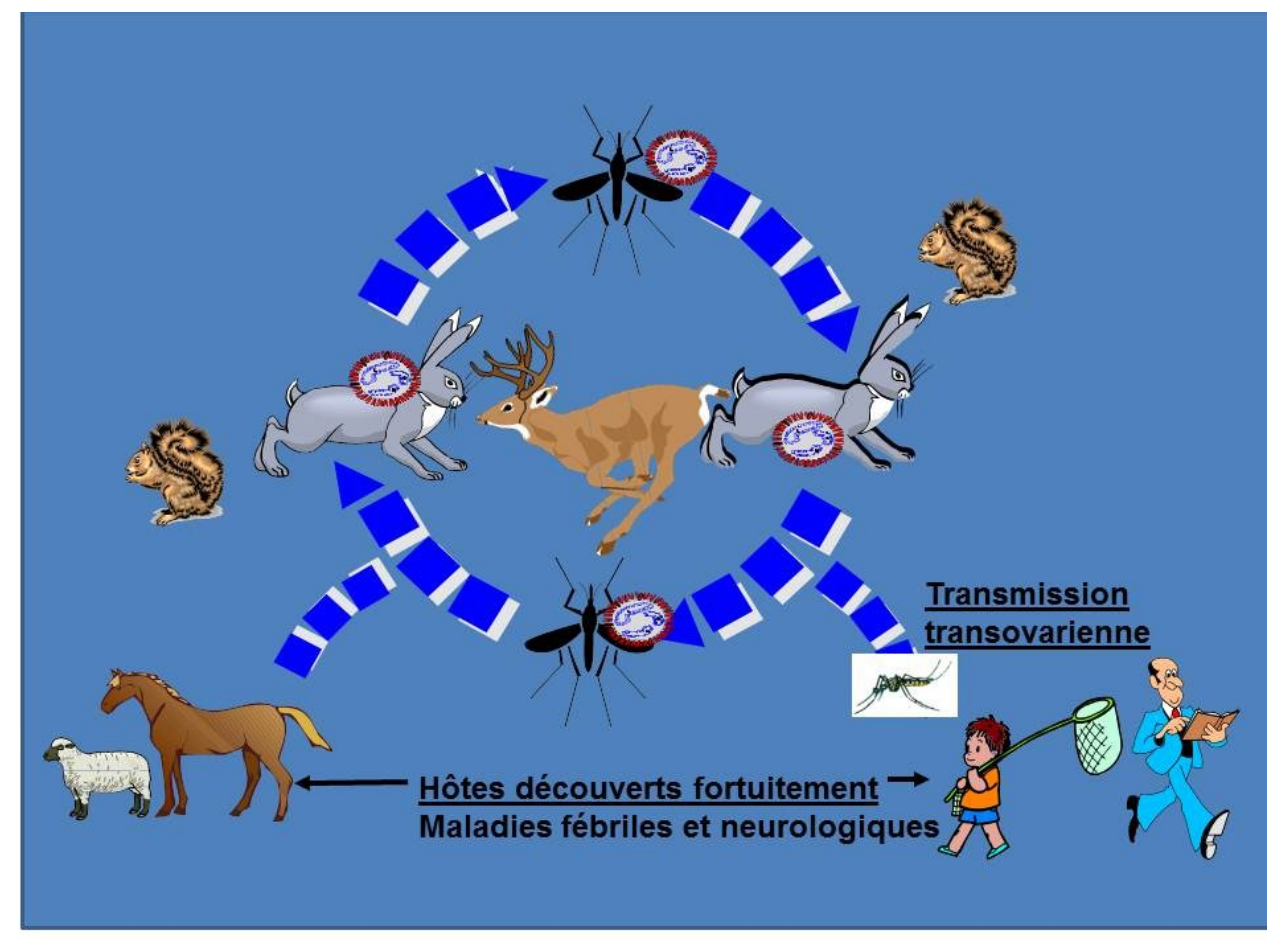

La présence de vecteurs et de réservoirs dans les régions boisées et les parcs des zones rurales et urbaines augmente la possibilité qu'il y ait des niveaux importants de circulation des virus non seulement dans les régions au sud du Canada, mais aussi dans les régions au nord, comme le Yukon, les Territoires du Nord-Ouest et l'Alaska. Par conséquent, il existe un risque d'exposition des humains et des animaux pendant toute la saison des moustiques, et ce, dans une vaste zone géographique. 


\section{Virus snowshoe hare}

Le virus snowshoe hare circule partout au Canada et aux États-Unis aux cycles enzootiques impliquant des moustiques qui n'appartiennent pas au genre Culex et des mammifères tels que les lièvres et les écureuils (8). II a été isolé pour la première fois en 1958 à partir du sérum d'un lièvre d'Amérique (Lepus americanus) au Montana. La maladie humaine causée par ce virus a été initialement observée au Canada en 1978 lorsque trois infections encéphalites ont été diagnostiquées au Québec (trois garçons âgés de 7, 6 et 10 ans présentant des symptômes : fièvre, nausées, vomissements, céphalées, confusion et agitation) et un cas de méningite relevé en Ontario (homme âgé de 30 ans) (8). La plupart des cas de virus snowshoe hare ont été associés à des maladies neuro-invasives telles que l'encéphalite et la méningite et touchaient principalement des enfants, avec une épidémiologie similaire à celle qui est observée pour le virus de La Crosse qui est étroitement lié et qui est présent à l'est et au centre-ouest des États-Unis (13).

\section{Virus de Jamestown Canyon}

Le virus de Jamestown Canyon a été isolé pour la première fois aux États-Unis en 1961 à partir d'un bassin de moustiques Culiseta inornata recueillis dans le Jamestown Canyon près de Boulder, au Colorado (4). Les infections par le virus de Jamestown Canyon peuvent être à l'origine d'un éventail de maladies similaire à celui observé pour le virus snowshoe hare, y compris l'infection fébrile et aiguë du système nerveux central $(4,7)$. Une affection du système respiratoire a été observée chez un certain nombre de patients. Contrairement au virus snowshoe hare, la plupart des personnes infectées par le virus de Jamestown Canyon présentant une maladie symptomatique grave sont des adultes et leurs principaux réservoirs se révèlent être les cerfs et les ruminants (7).

On a d'abord jugé que le virus de Jamestown Canyon était principalement concentré dans l'est de l'Amérique du Nord, mais des rapports récents laissent entendre que la répartition géographique des infections par le virus de Jamestown Canyon est plus large que celle reconnue auparavant (7). Selon les enquêtes sérologiques menées au Canada et aux États-Unis, on estime qu'environ $25 \%$ de la population pourrait avoir des anticorps contre le virus de Jamestown Canyon, mais les taux réels de séroprévalence peuvent aller de $1 \%$ à $40 \%$, voire plus, pour le virus snowshoe hare et le virus de Jamestown Canyon, selon la région $(22,23,24$, données non publiées de Drebot). Le rapport infections asymptomatiques/infections symptomatiques se situe environ entre 100:1 à 1500:1 d'après des études menées sur le virus connexe de La Crosse (25). De nombreuses infections humaines par le virus de Jamestown Canyon peuvent ne pas être détectées en raison de son évolution clinique non spécifique et de la disponibilité limitée de tests sensibles pour l'agent.

\section{Symptômes cliniques}

Les symptômes les plus fréquents comprennent les céphalées, la fièvre, les vertiges et les vomissements, et on peut également observer une photophobie, une détresse respiratoire et des éruptions cutanées $(25,26)$. Lorsque le système nerveux central est affecté, des syndromes cliniques allant de céphalées fébriles, de faiblesses musculaires à la méningite à liquide clair et à l'encéphalite peuvent survenir; il est en général impossible de les distinguer de syndromes semblables causés par d'autres virus. La méningite virale du sérogroupe California se caractérise par de la fièvre, des céphalées, une raideur de la nuque et de la pléocytose, et les infections peuvent entraîner des convulsions chez les enfants. L'encéphalite associée au virus snowshoe hare ou au virus de Jamestown Canyon se caractérise par de la fièvre, des céphalées et une altération de l'état mental allant de confusion au coma, avec ou sans autres signes de dysfonctionnement cérébral $(7,8,26,27)$. Les graves infections du sérogroupe California entraînent diverses séquelles, telles que des changements de comportement, des difficultés d'apprentissage et des déficits cognitifs $(25,26)$.

\section{Diagnostic en laboratoire}

Les procédures sérologiques pour les virus du sérogroupe California telles que le test d'immuno-absorption enzymatique (ELISA) révélateur d'anticorps IgM et le test de séro-neutralisation par réduction des plages sont les principales méthodes de dépistage utilisées pour diagnostiquer les infections par des virus du sérogroupe California $(7,11,12,13)$. Il est recommandé d'utiliser les sérums en phase aiguë et en phase convalescente issus de cas suspects pour déterminer le diagnostic d'augmentation ou de diminution des titres d'anticorps propres au 
sérogroupe California et consigner les séroconversions. Pour les cas de maladie neurologique, des échantillons de liquide céphalorachidien doivent être inclus aux fins de détection d'anticorps IgM aigus ou de séquences génomiques virales (par réaction en chaîne de la polymérase) qui constitueraient également une preuve confirmative d'infection avec les caractéristiques cliniques connexes $(11,12,26)$. Toutefois, il convient de noter qu'il est plutôt rare de détecter le virus snowshoe hare ou le virus de Jamestown Canyon à la fois dans le tissu issu de la biopsie cérébrale et dans le liquide céphalorachidien par réaction en chaîne de la polymérase ou par isolement (26).

Comme cela a été observé pour les infections par le virus du Nil occidental, il a été démontré que les anticorps IgM peuvent persister pendant plusieurs mois voire pendant des années dans le sérum de patients exposés à un virus du sérogroupe California (27). Par conséquent, les anticorps IgM persistants peuvent entraîner une certaine confusion quant aux diagnostics utilisés pour déterminer les cas actuels de maladie liée au sérogroupe California lorsqu'une sérologie positive est observée en utilisant uniquement des échantillons de sérum en phase aiguë.

\section{Définitions de cas pour les virus du sérogroupe California (virus snowshoe hare et virus de Jamestown Canyon)}

Un cas « confirmé » se fonde sur les critères de laboratoire suivants :

- Multiplication par quatre ou plus des titres d'anticorps propres au virus

- Présence d'anticorps IgM propres au virus ou d'anticorps de neutralisation dans le liquide céphalorachidien

- Détection d'ARN propre au virus dans le liquide céphalorachidien ou isolement du virus par culture cellulaire (rarement observé)

Un cas « probable » comprend:

Une personne ayant une maladie compatible sur le plan clinique (et des symptômes observés au cours de la saison des moustiques) ainsi que des anticorps IgM détectables contre le virus snowshoe hare ou le virus de Jamestown Canyon et des anticorps de neutralisation propres au sérogroupe California dans l'échantillon de sérum en phase aiguë.

\section{Surveillance en laboratoire}

Le Laboratoire national de microbiologie a élaboré des plateformes sérologiques pour tester les infections du sérogroupe California révélateur d'anticorps IgM et test de séro-neutralisation par réduction des plages en 2005 , après une période où il n'y a eu aucune procédure de diagnostic permettant de déterminer la présence de ces agents pathogènes $(9,11)$. Grâce à l'intégration de ces essais aux fins de dépistage des cas suspects d'agents transmis par les moustiques autres que le virus du Nil occidental, la première infection par virus du sérogroupe California en plus de 15 ans concernait un cas de virus snowshoe hare pédiatrique en Nouvelle-Écosse en 2006 (9).

Depuis 2006, plus de 200 cas " probables » et « confirmés " d'infections par le virus snowshoe hare et le virus de Jamestown Canyon ont été observés, y compris des cas confirmés de maladie neurologique dans diverses provinces dans tout le Canada (11,12, Drebot, résultats non publiés). Les bilans cliniques et diagnostiques détaillés ainsi que les identifications des cas concernaient un patient en Colombie-Britannique, trois patients en Alberta, un patient au Manitoba, un patient au Québec, un patient au Nouveau-Brunswick et un patient en Nouvelle-Écosse (10, Drebot, résultats non publiés). La majorité des cas probables et confirmés ont été associés au virus de Jamestown Canyon (70 \%), ce qui va à l'encontre de ce qui a été observé au cours des années 1970 et 1980, où la plupart des cas d'infections virales au Canada du sérogroupe California étaient attribués au virus snowshoe hare (8). On ne sait pas avec certitude si cela est dû à un changement relatif à la circulation ou à l'abondance du virus ou si cela est dû à l'amélioration de la sensibilité et de la spécificité des diagnostics sérologiques. Grimstead et al. ont indiqué que les épreuves d'inhibition de l'hémagglutination utilisées précédemment pourraient ne pas être assez sensibles pour détecter les expositions au virus de Jamestown Canyon par rapport aux méthodes sérologiques actuellement utilisées telles que le test d'immuno-absorption enzymatique (ELISA) révélateur d'anticorps IgM (24). La prépondérance du virus de Jamestown Canyon dans les 
récentes enquêtes sérologiques concorde également avec le fait que ce virus est l'agent du sérogroupe California auquel la plupart des personnes au Canada sont exposées $(11,12,22,23$, Drebot, résultats non publiés).

\section{Virus de Cache Valley}

Le virus de Cache Valley est un autre Orthobunyavirus qui est principalement transmis par les moustiques n'appartenant pas au genre Culex. Comme pour le virus de Jamestown Canyon, on pense que son principal réservoir animal ou hôte amplificateur est le cerf $(15,16)$. Le virus de Cache Valley a été isolé pour la première fois en 1956 à Cache Valley, dans l'Utah, aux États-Unis, mais il est endémique dans tout le Canada, les États-Unis, les Caraïbes, le Mexique et l'Argentine $(15,28,29)$. Bien que le virus de Cache Valley puisse infecter les humains ainsi qu'un large éventail d'animaux de bétail, la maladie clinique a été principalement observée chez les moutons (15). La plupart des infections naturelles chez les moutons (non en gestation) sont subcliniques; toutefois, le virus peut traverser le placenta chez les brebis gravides et infecter le fœtus, ce qui entraîne la naissance d'agneaux malades ayant des malformations observées au niveau de l'appareil locomoteur et du système nerveux central. Par le passé, le virus de Cache Valley a été soupçonné d'être à l'origine des flambées épidémiques qui ont eu lieu chez les moutons au Canada en raison de la sérologie positive des brebis des troupeaux fermiers, mais, en 2012 et 2013, les infections de bétail par le virus de Cache Valley ont été vérifiées par isolement viral et réaction en chaîne de la polymérase positive dans les tissus pour la première fois en Ontario et au Québec $(30,31)$. Les études de séroprévalence ont révélé des taux de séropositivité pouvant atteindre $40 \%$ chez les moutons et d'autres ruminants à différents endroits de la Saskatchewan, de l'Ontario et du Québec.

\section{Cas cliniques}

Les infections humaines par le virus de Cache Valley semblent être assez courantes dans les régions où le virus est enzootique et où les taux de séroprévalence chez les humains peuvent être atteindre $18 \%(28)$. Bien qu'une maladie neuro-invasive humaine ait rarement été diagnostiquée, il y a eu trois cas de maladie grave associée au virus de Cache Valley aux États-Unis, y compris un cas mortel d'encéphalite $(18,19)$. La faible fréquence des cas est vraisemblablement due au fait que les laboratoires dépistent rarement le virus et que les cas impliquant une maladie fébrile et neuro-invasive peuvent ne pas être diagnostiqués. Le dépistage sérologique des cas suspects de virus du Nil occidental au Manitoba et en Saskatchewan a permis d'identifier l'exposition au virus de Cache Valley chez 5 à $16 \%$ des patients soumis à un test de détection des anticorps propres au virus (17). Ainsi, la souche du virus de Cache Valley isolée à partir d'un cas humain récent aux États-Unis était presque identique aux isolats obtenus pendant les flambées épidémiques qui ont eu lieu chez les moutons au Québec et en Ontario, ce qui indique que des génotypes du virus actuellement en circulation peuvent présenter un risque de

pathogénicité chez les humains et d'autres animaux (30). Le virus de Cache Valley virus a également été associé à des anomalies congénitales chez les humains ( $p$. ex. macrocéphalie chez les enfants en bas âge), mais le rôle précis que peut jouer le virus de Cache Valley dans l'induction de ces anomalies n'a pas été déterminé et d'autres vérifications sont nécessaires (32).

\section{Diagnostic en laboratoire}

Aucune trousse de diagnostic commerciale n'est disponible pour identifier les cas de virus de Cache Valley et les anticorps contre l'agent n'auront pas une réaction croisée importante dans le cadre des essais relatifs au sérogroupe California. Le Laboratoire national de microbiologie (LNM) effectue des tests de neutralisation pour déterminer la présence d'anticorps propres au virus de Cache Valley dans le sérum et a également mis en place des procédures d'isolement (17). Des essais immuno-enzymatique (ELISA) révélateurs d'anticorps IgM et lgG sont actuellement élaborés pour le virus de Cache Valley afin de faciliter la détection des cas. Compte tenu de la diversité de l'agent pathogène potentiel au Canada et de l'identification croissante de cas chez le bétail au Canada, le potentiel de cas neuro-invasifs chez les patients au cours de la saison des moustiques est faible mais existant. 


\section{Discussion}

Les bunyavirus transmis par les moustiques qui sont nouveaux et émergents, comme les virus du sérogroupe California et le virus de Cache Valley, peuvent contribuer à un nombre important de cas de maladie fébrile et neuro-invasive non diagnostiquée au cours de la saison des moustiques au Canada. La récente observation de cas de virus de Jamestown Canyon et de virus snowshoe hare indiquent que ces virus contribuent actuellement à une morbidité importante lorsque les moustiques sont prévalents. Une récente étude de Kulkarni et al. (33) a utilisé des statistiques spatiales et temporelles pour déterminer les grappes saisonnières d'hospitalisations canadiennes et a laissé entendre que les agents arboviraux, outre le virus du Nil occidental, pourraient être impliqués en tant qu'étiologies non déterminées de maladies neurologiques. Il est à noter que le risque saisonnier et géographique d'exposition aux bunyavirus transmis par les moustiques est plus important que celui d'exposition au virus du Nil occidental.

II est probable qu'un nombre important d'infections par bunyavirus transmis par les arthropodes ne soit pas détecté en raison de l'absence d'essais diagnostiques disponibles au niveau commercial et du faible niveau de surveillance. À l'heure actuelle, le Laboratoire national de microbiologie est le seul laboratoire au Canada à effectuer le dépistage des virus du sérogroupe California. Il existe un seul test sérologique commercial pour les virus du sérogroupe California, et il est principalement utilisé en tant que test d'immunofluorescence des anticorps contre le virus de La Crosse (20). Bien qu'une réactivité sérologique croisée entre le virus snowshoe hare, le virus de Jamestown Canyon et le virus de La Crosse peut se produire, des études récentes ont révélé que les plateformes de diagnostic propres au virus de La Crosse ne parviennent pas toujours à détecter les anticorps contre d'autres virus du sérogroupe California et il se peut que certains cas ne soient pas repérés (20). La mise au point et la mise en œuvre de trousses commerciales et « internes » pour un éventail plus important d'orthobunyaviruses pourraient aider à détecter un nombre supérieur de cas associés à ces virus. Des diagnostics améliorés et réalisés en temps opportun aideraient les cliniciens à prendre des décisions en matière de soins et de prise en charge des patients.

La prévention de la maladie se fait principalement par l'éducation du public. Les mesures de réduction des risques personnels comprennent la diminution du risque de piqûres de moustiques en évitant l'exposition, le port de vêtements protecteurs et l'utilisation d'un insectifuge. Il est également recommandé d'éliminer les sites de reproduction des moustiques pour prévenir l'infection à arbovirus.

\section{Conclusion}

Une maladie fébrile et neurologique peut être causée par des bunyavirus transmis par les moustiques émergents au Canada. Les cliniciens devraient envisager les infections par les virus du sérogroupe California et le virus de Cache Valley dans les diagnostics différentiels lorsqu'on soupçonne la présence d'une infection à arbovirus et que le dépistage du virus du Nil occidental n'est pas concluant. Une surveillance accrue et l'utilisation d'un plus large panel de méthodes de diagnostic pourraient permettre de mieux identifier les maladies neuro-invasives causées par ces virus émergents. Entre-temps, il est recommandé d'éduquer le public en ce qui concerne ces virus et de souligner l'importance de prendre des mesures de prévention personnelles pour diminuer le risque d'infection.

\section{Remerciements}

L'auteur tient à remercier pour leur aide les laboratoires de santé publique et les laboratoires provinciaux vétérinaires du Canada qui ont pris part à l'identification des cas bunyavirus associés aux virus du sérogroupe California ou au virus de Cache Valley. L'auteur remercie également Harvey Artsob, Ph. D, pour avoir partagé les résultats non publiés concernant les cas de virus du sérogroupe California, ainsi que Kai Makowski, Kristina Dimitrova, Kimberly Holloway et Maya Andonova pour leur excellente expertise technique et leurs contributions au dépistage. 


\section{Conflit d'intérêts}

Aucun.

\section{Références}

(1) King AMQ, Lefkowitz E, Adams MJ, Carstens EB. Family bunyaviridae. In: King AMQ, Adams MJ, Carstens EB, Lefkowitz EJ, editors. Virus Taxonomy: Ninth Report of the International Committee on Taxonomy of Viruses. San Diego: Elsevier; 2012. p. 725-741.

(2) Kruger DH, Figueiredo LT, Song JW, Klempa B. Hantaviruses--globally emerging pathogens. J Clin Virol. 2015 Mar; 64:128-36.

(3) Drebot MA, Jones S, Golla A, Safronetz D, Strong JE, Kobinger G, Lindsay RL. Hantavirus pulmonary syndrome in Canada: An overview of clinical features, diagnostics, epidemiology, and prevention. Can Commun Dis Rep. 2015 June;41(6).

Disponible en français: Le syndrome pulmonaire à Hantavirus au Canada : Aperçu du tableau clinique, des diagnostics, de l'épidémiologie et de la prévention

(4) Leduc JW. Epidemiology and ecology of the California serogroup viruses. Am J Trop Med Hyg. 1987;37(3) Suppl: 60S-68S.

(5) Putkuri N, Kurkela S, Levanov L, Huhtamo E, Vaheri A, Sironen T, Vapalahti O. Isolation and characterization of a California encephalitis serogroup Othobunyavirus from Finnish mosquitoes. Infect Genet Evol. 2014;22:164-73.

(6) Lindsey NP. Lehman JA.Staples JE. Fischer M. West Nile virus and other arboviral diseases - United States, 2013. MMWR. 2014;63(24):521-526.

(7) Lowell J, Higgins DP, Drebot M, Makowski K, Staples E. Human Jamestown Canyon virus infection --- Montana 2009. MMWR. 2011;60(20):652-655.

(8) Artsob H. Arbovirus activity in Canada. Arch Virol. 1990;(Suppl 1):249-58.

(9) Meier-Stephenson V, Langley JM, Drebot M, Artsob H. Encephalitis in the summer: A case of snowshoe hare (California serogroup) virus infection in Nova Scotia. CAN COMMUN DIS REP. 2007;33(11):23-6.

(10) Drebot M.A. A laboratory-confirmed case of Jamestown Canyon virus encephalitis in a Quebec resident with travel history to Maine and New Hampshire. Am J Trop Med Hyg. 2012;87(5)(Suppl 1):280.

(11) Makowski K, Dimitrova K, Andonova M, Drebot M. An overview of California serogroup virus diagnostics and surveillance in Canada in 2008. Int J Antimicrob Agents. 2009;34:S19.

(12) Makowski K, Dimitrova K, Andonova M, Holloway K, Kadkhoda K, Van Caeseele P, Drebot M. The identification of probable cases of California serogroup virus infections in Manitoba in 2010. Can J Infect Dis Med Microbiol. 2011;22:15A-6A.

(13) Gaensbauer JT, Lindsey NP, Messacar K, Staples JE, Fischer M. Neuroinvasive arboviral disease in the United States: 2003-2012. Pediatrics. 2014;134(3);e642-650.

(14) Mechai S, Margos G, Feil EJ, Lindsay LR, Ogden NH. Recent and projected future climatic suitability of North America for the Asian tiger mosquito Aedes albopictus. Appl Environ Microbiol. 2014 Dec 12. pii: AEM.03730-14. [Epub ahead of print].

(15) de la Concha-Bermejillo, A. Cache Valley virus is a cause of fetal malformation and pregnancy loss in sheep. Small Ruminant Res. 2003;49:1-9.

(16) Andreadis TG et al. 2014 Spatial-temporal analysis of Cache Valley virus (Bunyaviridae: Orthobunyavirus) infection in Anopheline and Culicine mosquitoes (Diptera: Culicidae) in the Northeastern United States, 1997-2012. Vector Borne Zoonotic Dis. 2014;14(10):763-773.

(17) Dimitrova K, Andonova M, Makowski K, Holloway K, Levett PN, Kadkhoda K, Drebot M. Preliminary evidence of Cache Valley virus infections and associated human illness in western Canada in 2009. Can J Infect Dis Med Microbiol. 2011;22:15A

(18) Sexton DJ, Rollin PE, Breitschwerdt EB, et al. Life-threatening Cache Valley virus infection. N Engl J Med. 1997;336:547-9.

(19) Nguyen NL, Zhao G, Hull R, Shelly MA, Wong SJ, Wu G et al. Cache Valley virus in a patient diagnosed with aseptic meningitis. J Clin Microbiol. 2013;51:1966-9.

(20) Makowski K, Dimitrova K, Andonova M, Drebot M. Assessing serological cross-reactivity among California serogroup viruses using an IgM ELISA platform. Can J Infect Dis Med Microbiol. 2010;21:26A.

(21) Goff G, Whitney H, Drebot MA. Roles of host species, geographic separation and isolation in the seroprevalence of Jamestown Canyon and snowshoe hare viruses in Newfoundland. Appl Environ Microbiol. 2012 Sep;78(18):6734-40. Adjemian J, Weber IB, Quiston J, et al. Zoonotic infections among employees from Great Smoky Mountains and Rocky Mountain National Parks, 2008-2009. Vector Borne and Zoonotic Dis. 2012;12(11):922-931.

(23) Sampasa-Kanyinga H, Levesque B, Anassour-Laouan-Sidi E, et al. Zoonotic infections in communities of the James Bay Cree territory: An overview of seroprevalence. Can J Infect Dis Med Microbiol. 2013;24(2):79-84. 
(24) Grimstad PR, Calisher CH, Harroff RN, Wentworth BB. Jamestown Canyon virus (California serogroup) is the etiological agent of widespread infection in Michigan humans. Am J Trop Med Hyg. 1986;35(2):376-386.

(25) Haddow AD, Odol A. The incidence risk, clustering and clinical presentation of La Crosse virus infections in eastern United States, 2003 - 2007. PLOS One. 2009;4(7):e6145.

(26) Huang C. Campbell W. Grady L. Kirouac I. LaForce FM. Diagnosis of Jamestown Canyon encephalitis by polymerase chain reaction. Clin Infect Dis. 1999;28:1294-7.

(27) Makowski K, Dimitrova K, Andonova M, Vancaeseele P, Dawood M, Drebot M. IgM persistence: A diagnostic concern for identifying cases of California serogroup virus infection. Can J Infect Dis Med Microbiol. 2010;21:26A-7A.

(28) Blitvich BJ, Saiyasombat R, Talavera-Aguilar LG, Garcia-Rejon JE, Farfan-Ale JA, Machain-Williams C, Loroño-Pino MA. Orthobunyavirus antibodies in humans, Yucatan Peninsula, Mexico. Emerg Infect Dis. 2012;18(10):1629-1632.

(29) Blitvich BJ, Saiyasombat R, Travassos da Rosa A, Tesh RB, Calisher CH, Garcia-Rejon JE, Farfán-Ale JA, Loroño $\mathrm{RE}$, Bates A, Loroño-Pino MA. Orthobunyaviruses: A common cause of infection of livestock in the Yucatan peninsula of Mexico. Am J Trop Med Hyg. 2012 Dec;87(6):1132-9.

(30) Shapiro J, Brooks A, Menzies P, Rau J, Drebot M, Andonova M et al. Cache Valley virus identified as a cause of malformed lambs in Ontario. AHL News. 2012;16:15.

(31) MAPAQ. Cas de malformations congenitales chez des ovins causees par le virus de la Vallee Cache. Info-RAIZO. 2013;1.

(32) Calisher $\mathrm{CH}$, Sever JL. Are North American bunyamwera serogroup viruses etiologic agents of human congenital defects of the central nervous system? Emerg Infect Dis. 1995 Oct-Dec;1(4):147-51.

(33) Kulkarni MA,Lecocq AC. Artsob H. Drebot MA. Ogden NH. Epidemiology and aetiology of encephalitis in Canada, 1994-2008: A case for undiagnosed arboviral agents? Epidemiol Infect. 2013;141:2243-2255. 